MATEC Web of Conferences 21, 11002 (2015)

DOI: $10.1051 /$ matecconf $/ 20152111002$

(C) Owned by the authors, published by EDP Sciences, 2015

\title{
Effective electromagnetic forces in thin sheet metal specimen
}

\author{
L. Langstädtler ${ }^{\mathrm{a}}$, C. Schenck, and B. Kuhfuss \\ Bime, Bremen Institute for Mechanical Engineering, University of Bremen, Germany
}

\begin{abstract}
Electromagnetic forming is mainly investigated for the macro world as the body forces in this high speed process are decreasing with the volume of the specimen. For micro metal sheets different effects are observed which make an analysis of the acting forces more difficult. Hence, the validity of process simulations for electromagnetic forming is still limited. In this research the effective electromagnetic force on thin EN AW-1050A (A199.5) sheet metals is investigated by varying the loading energy $E_{C}$, the ration $s_{R}$ between sheet thickness and skin depth, the sheets width $b$ and the distance $d_{c}$ between passive tool and sheet metal.
\end{abstract}

\section{Background}

Electromagnetic forming is a high speed forming method where one part of the tool is replaced by a strong pulsed electromagnetic field. In the process a high current peak $\mathrm{I}_{\max }$ in a tool coil induces eddy currents in an electrical conductive workpiece and accelerates it by repulsive body forces $\mathrm{F}_{\mathrm{L}}$ (Lorentz' forces) (see Fig. 1).

The current peak is generated by a LC-resonator. The process energy $E_{C}$ of the capacitor is influenced by its capacity $\mathrm{C}$ and the loading voltage $\mathrm{U}_{0}$, Eq. (1).

$$
E_{C}=0.5 C U_{0}^{2} .
$$

According to the skin effect the induced eddy currents are limited to the skin depth $\delta$. Depending on the coil, the workpiece can be shaped axis-symmetrically or planar and be used for different manufacturing processes like forming, embossing, cutting and joining. A technical advantage of electromagnetic forming is the high deformation rate (typical 2500 1/s [1]) which leads to higher plasticity. By replacing one part of the tool by contactless working forces there are no clearances between tool parts and the risk of abrasion decreases. The economical advantages of electromagnetic forming are the short cycle times and the lower tool costs. For electromagnetic forming the body forces $\mathrm{f}$ are depending on the current density $\mathrm{J}$ in the sheet and the magnetic field density B, Eq. (2).

$$
\mathbf{f}=\mathbf{J} \times \mathbf{B}=1 / \mu(\operatorname{rot} \mathbf{B}) \times \mathbf{B} .
$$

\footnotetext{
${ }^{a}$ Corresponding author: langstaedtler@bime.de
}

This is an Open Access article distributed under the terms of the Creative Commons Attribution License 4.0, which permits unrestricted use, distribution, and reproduction in any medium, provided the original work is properly cited. 


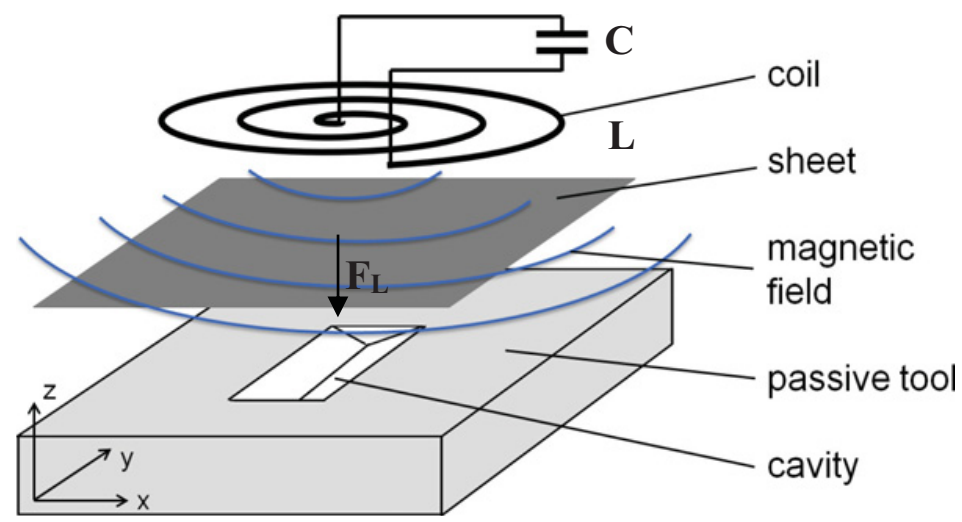

Figure 1. Scheme of electromagnetic forming.

The current density is influenced by the skin effect that limits the current to the skin depth $\delta$ depending on the rise time of the current. Hence, if the sheet thickness $\mathrm{s}_{0}$ is lower than the skin depth the body forces are limited by $\mathrm{s}_{0}$. Hence, electromagnetic forming is widely investigated for sheet metals and cylindrical workpieces with a thickness $\mathrm{s}_{0}$ of more than $1 \mathrm{~mm}$ [2]. Though electromagnetic forming is introduced to micro forming in special regard to the advantages like higher plasticity and simple tools without narrow tolerances [3-5]. The challenge of electromagnetic forming thin sheet metals is to compensate the decrease of body forces with decreasing sheet volume. Due to the skin effect the rise time $t_{r}$ of the current peak has to be very short to shield the magnetic field from diffusion through the sheet. The rise time is influenced by the capacity $\mathrm{C}$ and the inductivity $\mathrm{L}$ of the resonator. For a shorter rise time reducing $\mathrm{C}$ effects less energy and a lower peak current. Furthermore shortening the rise time by a smaller inductivity causes a reduction of the magnetic field intensity and in consequene the body forces decrease. Another challenge is the limitation of $\mathrm{I}_{\max }$ due to the local heating of the micro sheet by high eddy currents that can melt or even vaporize the material. At least the coil design has to be adapted to sheet metal micro forming due to its homogeneity and stiffness. An improvement for micro metal forming is a concentration of the magnetic field by a redesign of the tool system (coil and passive tool) [5]. To find optimal process parameter values the frequency was investigated in several studies by simulations and a counterproductive attracting force for thin metal sheets was observed for $\mathrm{s}_{0}<\delta$. The simulations were validated by measurements of the sheet metal deformation. Several investigations showed, that a previous recommendation for the ratio $s_{R}$ of 3 between sheet thickness and skin depth can be lowered, Eq. (3) [6-8].

$$
s_{R}=s_{0} / \delta .
$$

Investigations for electromagnetic micro forming of thin sheet metals showed that a forming, cutting and joining is possible. In this case losses by diffusion of the magnetic field through the sheet can be compensated by a higher magnetic pulse and a redesign of the coil and the passive tool for concentrating the magnetic field [5]. With these measures even micro metal sheets with a thickness of $10 \mu \mathrm{m}$ can be formed using cheaper tools, short cycle times and a better material behaviour. As the sheet is diffused by the electromagnetic field, the passive tool influences the magnetic field and with this the acting forces.

\section{Experiments}

In this research the effective force $F_{E}$ is investigated. The main motivation is to validate the effective force for different ratios $\mathrm{s}_{\mathrm{R}}$ for $\mathrm{s}_{\mathrm{R}}<3$ by numerical simulations and physical experiments. 


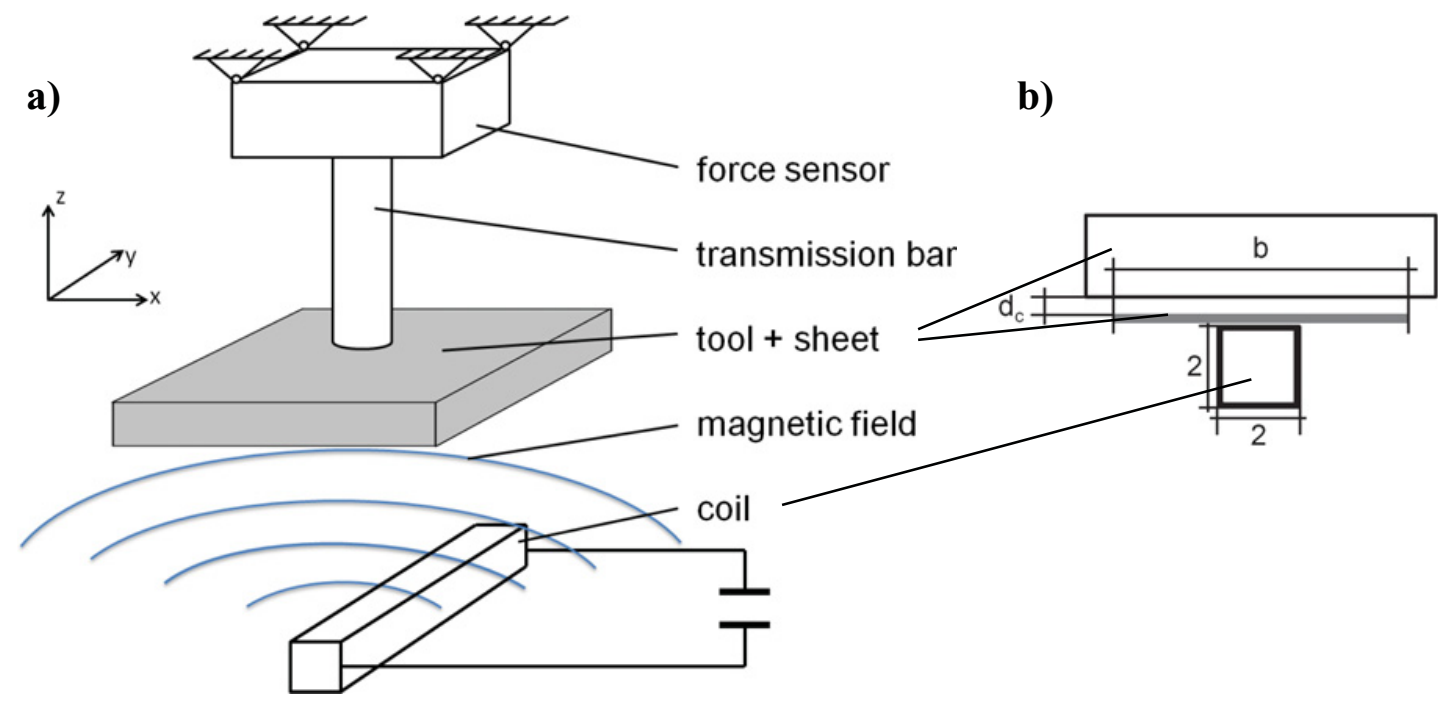

Figure 2. Experimental set up a) scheme of set up and b) cross section of tool, sheet and single inductor.

\subsection{Set up}

In the physical experiments A199.5 metal sheets are positioned under an electrical non conductible mounting plate which is connected to a load cell with an insulated transmission bar. Effects that make a validation difficult for thin sheets are minimized for example by not deforming the sheet (see Fig. 2). For the validation the distance between inductor and sheet has to be minimized. The lateral dimension of the sheets is $50 \times 50 \mathrm{~mm}^{2}$. The force is generated by a LC-resonator with a capacity $\mathrm{C}=100 \mu \mathrm{F}$, a maximum loading voltage $\mathrm{U}_{0}=6 \mathrm{kV}$, a maximum loading energy $\mathrm{E}_{\mathrm{C}}=1800 \mathrm{~J}$ and a maximum current pulse $\mathrm{I}_{\max }=70 \mathrm{kA}$. The inductance of the circuit is $\mathrm{L}_{\mathrm{T}}=0.5 \mu \mathrm{H}$, the natural frequency is $\mathrm{f}_{0}=22 \mathrm{kHz}$ and the current rise time is $t_{r}=11 \mu \mathrm{s}$. The discharge current is measured by a current transducer (Rogowski coil). A rectangular singe inductor with a cross-section of $2 \times 2 \mathrm{~mm}^{2}$ is used. This leads to shorter current rise times by a lower inductance.

The advantage of this simplified coil design is the homogeneous electromagnetic field and the resulting homogeneous force distribution. In Fig. 3 the oscillating current in LC-resonator and the force peak detected at the force sensor is shown. The force sensor and transmission bar is preloaded to reduce the elastic deformation. The force peak is delayed with $t_{\mathrm{d}}$ to the current peak. This delay is caused by the transmission behaviour of the experimental set up and the inertia of the sheet metal during the pulse transmission. The experimental set up shows a good repeatability of the transmission behaviour during the variation of process parameters. The varied parameters in this research are the loading energy $\mathrm{E}_{C}$ by the loading voltage $\mathrm{U}_{0}$. The sheets thickness $\mathrm{s}_{0}$ is varied to investigate the influence of sheet thickness and skin depth. Due to embossing experiments a clearance $\mathrm{d}_{\mathrm{c}}$ between the sheet and the tool has to be defined to accelerate the sheet to a velocity of $200 \mathrm{~m} / \mathrm{s}$ enhancing the embossing result due to its kinetic energy $[3,4,9]$. For this the effective force is measured varying $d_{c}$. Furthermore the width $b$ of the sheet is varied.

\subsection{Process simulations}

The process is simulated with an electromagnetic 2D-model, cf. Fig. 2b, using the discharge function of the measured current pulse. According to Paese et al. the electromagnetic simulations show an increase 


\section{MATEC Web of Conferences}

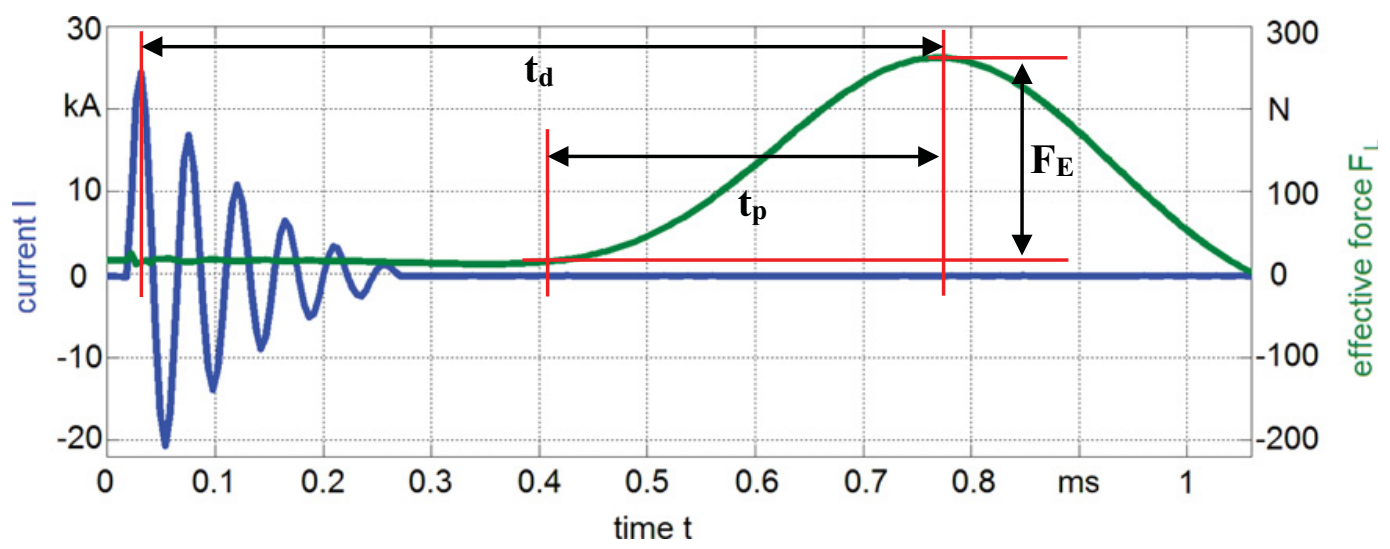

Figure 3. Measurement of the current pulse and the force peak; $\mathrm{s}_{0}=1.5 \mathrm{~mm}$ and $\mathrm{E}=200 \mathrm{~J}$.
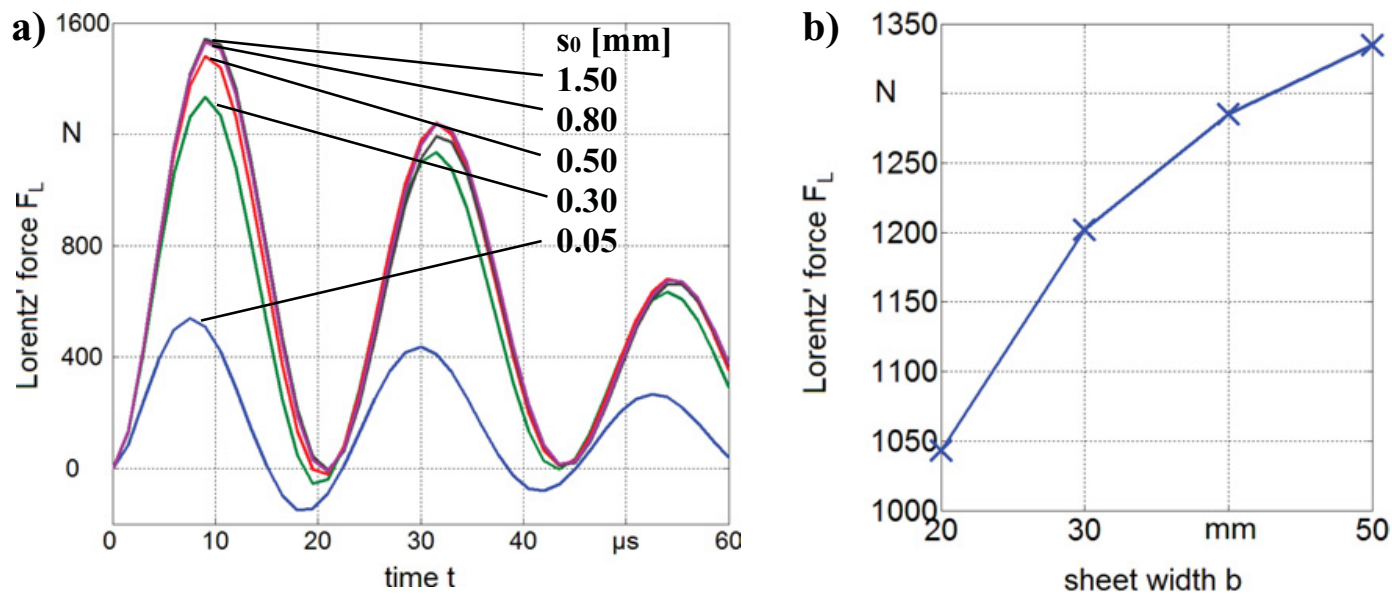

Figure 4. a) Electromagnetic simulations varying $\mathrm{s}_{0}$; single inductor $2 \times 2 \mathrm{~mm}^{2}, \delta=0.7 \mathrm{~mm}$, E = w200 J b) variation of the sheets width $\mathrm{b}$; single inductor $2 \times 2 \mathrm{~mm}^{2}, \mathrm{E}_{\mathrm{C}}=200 \mathrm{~J}$ (Lorentz' force at first peak).

of the Lorentz' force $\mathrm{F}_{\mathrm{L}}$ with increasing the sheet thickness $\mathrm{s}_{0}$, see Fig. $4 \mathrm{a}$. Due to an increasing resistance in the sheet an attracting force $\left(\mathrm{F}_{\mathrm{L}}<0\right)$ can be observed caused by a phase shifting between inducing current and eddy currents in the sheet [7].

A maximum value for the Lorentz' force is reached with a sheet thickness of about $1.5 \mathrm{~mm}$, but even with a ratio $s_{R}$, of 1 more than $95 \%$ of the maximum force is reached. Increasing the sheet thickness from this point, a thicker sheet $\left(\mathrm{s}_{0}>\delta\right.$ ) will have less influence on the force. Besides varying the sheet thickness also the width $b$ of the sheet influences the acting force, Fig. 4b. Increasing the width the Lorentz' force increase caused by enlarging the participating magnetic field.

\subsection{Parameter study}

In the following experimental parameter study the effective force is measured as shown in Fig. 3 varying the loading energy $\mathrm{E}_{\mathrm{C}}$ by $\mathrm{U}_{0}$, the sheet thickness $\mathrm{s}_{0}\left(\right.$ e.g. $\mathrm{s}_{R}$ ) and the clearance $\mathrm{d}_{\mathrm{c}}$. Increasing $\mathrm{E}_{\mathrm{C}}$ leads to an increase of the effective force $\mathrm{F}_{\mathrm{E}}$ (see Fig. 5). A higher energy leads to a higher current pulse which 


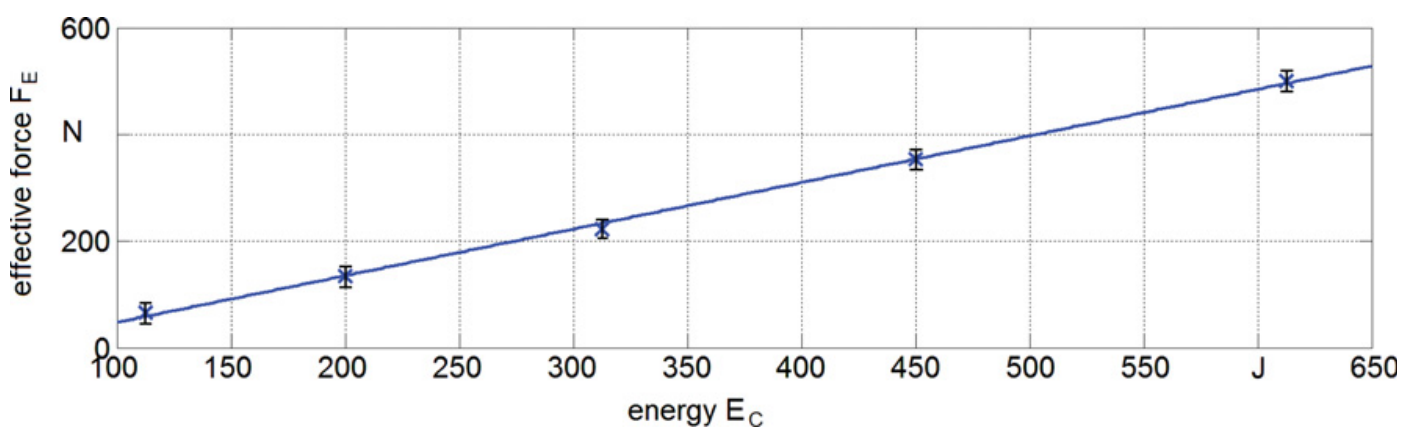

Figure 5. Variation of the energy $\mathrm{E}_{\mathrm{C}} ; \mathrm{b}=50 \mathrm{~mm}, \delta=0.7 \mathrm{~mm}, \mathrm{~s}_{0}=0.30 \mathrm{~mm}$.

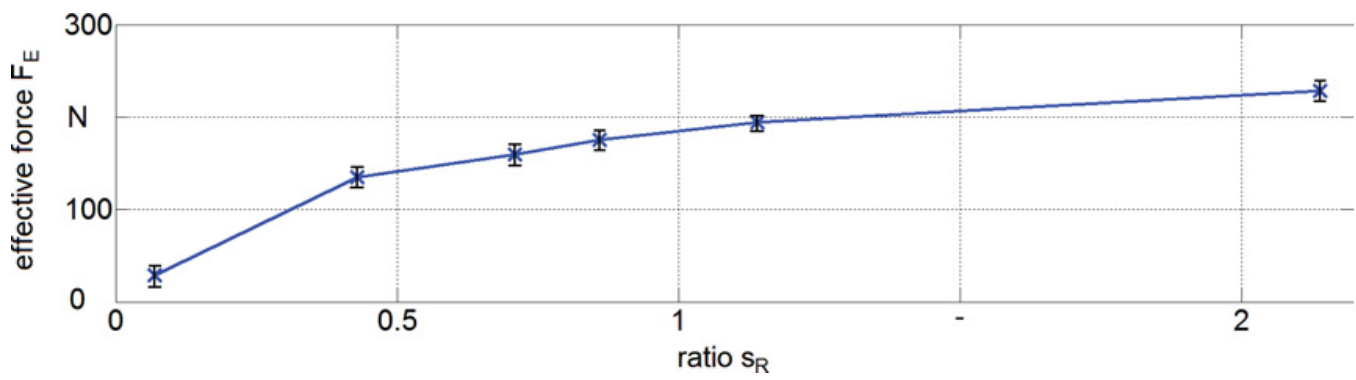

Figure 6. Variation of the ratio $\mathrm{s}_{\mathrm{R}}$ between sheet thickness and skin depth; $\mathrm{b}=50 \mathrm{~mm}, \delta=0.7 \mathrm{~mm}, \mathrm{E}_{\mathrm{C}}=200 \mathrm{~J}$.

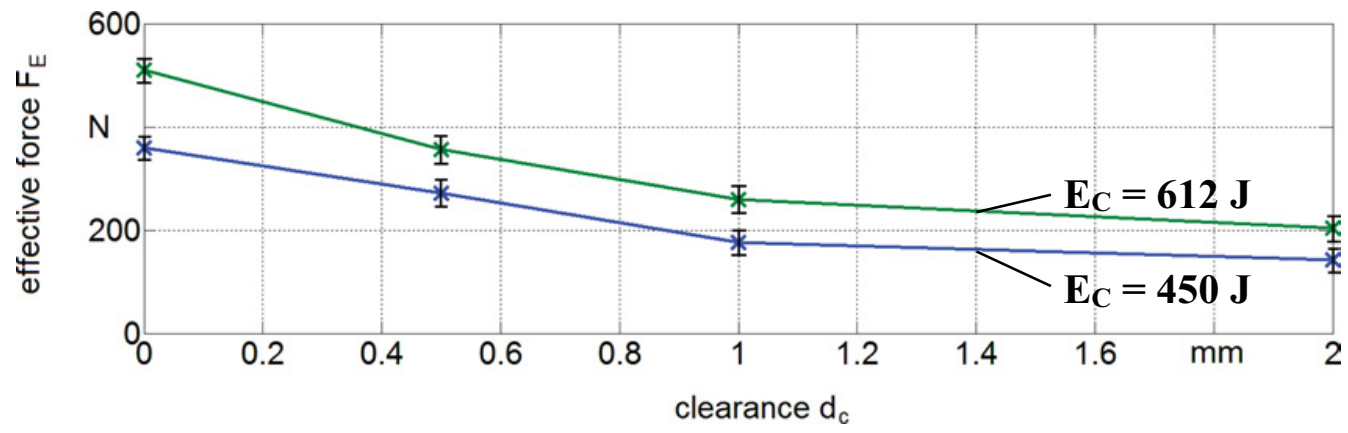

Figure 7. Variation of the clearance $\mathrm{d}_{\mathrm{c}} ; \mathrm{b}=50 \mathrm{~mm}, \delta=0.7 \mathrm{~mm}, \mathrm{E}_{\mathrm{C}}=450 \mathrm{~J}$ and $\mathrm{E}_{\mathrm{C}}=612 \mathrm{~J}, \mathrm{~s}_{0}=0.30 \mathrm{~mm}$.

directly influences the strength of the magnetic field and the induced eddy currents. According to Eq. (2) this results in a higher force in the sheet.

The variation of the sheet thickness $\mathrm{s}_{0}$, seen in Fig. 6, points out that the acting force on the sheet is limited to a maximum value. This correlates with the electromagnetic simulations. As the eddy currents in the sheet are limited to the skin depth, the body forces can only act on the eddy currents which leads to a maximum value at $\mathrm{s}_{0}=\delta$.

The investigations for electromagnetic embossing of optical micro structures showed, that an optimum clearance $d_{c}$ leads to an increase of the embossing result [9]. The variation of the clearance indicates a decrease of the effective force increasing the clearance $d_{c}$, Fig. 7. For a lower energy this characteristic is the same as for a higher energy. 


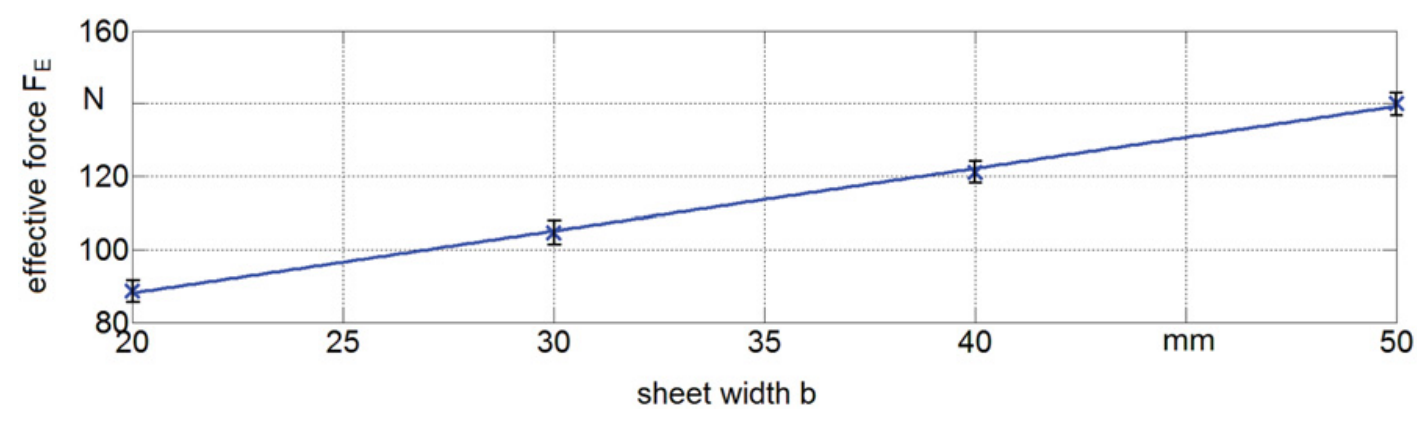

Figure 8. Variation of the sheets width $\mathrm{b} ; \delta=0.7 \mathrm{~mm}, \mathrm{E}_{\mathrm{C}}=200 \mathrm{~J}, \mathrm{~s}_{0}=0.30 \mathrm{~mm}$.

In Fig. 8 the effective force is shown for a different sheet width $b$. The effective force is increasing with the sheets width. This correlates with the electromagnetic simulations. At least for electromagnetic micro forming this effect has to be observed. A major challenge in the micro part production is the handling. An approach to overcome the problems is the production in linked parts. The parts are kept interconnected during the whole production process. An example is a stretch drawn cup which remains in the base material to be continuously fed [10]. As the forming of cups in the base material is complicated for conventional processes, the base material is advantageous for electromagnetic forming by an increased force and a also higher plasticity.

\section{Conclusion}

In this research a good qualitative match of the process simulations and the physical experiments can be observed. While the process simulation shows an oscillating force, the measured effective force is a peak resulting from the oscillation of repulsive and attracting forces.

The variation of the sheet thickness shows, that the previous recommendations for a ratio of $s_{R}$ of 3 can be lowered to $s_{R} \geq 1$. As it could be shown the width $b$ of the sheet can be used to increase the forming force for micro metal forming. One reason for the decrease of effective forces increasing the clearance $d_{c}$ can be an air cushion between sheet and passive tool. An increase of the embossing result can be caused by for example a change of material behaviour and an increase of plasticity while embossing which can't be observed measuring the force. This will be investigated in future work.

In summary this research shows that the effective force while electromagnetic forming can be investigated with the introduced set up. This set up will be used for following researches to validate the influence of the tool system (coil and passive tool) on the electromagnetic forces.

\section{References}

[1] Y.Y. Chu et al., Journal of Materials Processing 212: 1314-1323 (2012)

[2] V. Psyk et al., Journal of Materials Processing Technology 211: 787-829 (2011)

[3] Q. Zhao et al., Trans. Nonferrous Met. Soc. China 21: 461-464 (2011)

[4] M. Kamal et al., Journal of Materials Processing Technology 190, 41-50 (2007)

[5] B. Kuhfuss et al., Procedia Engineering 81: 2135-2140 (2014)

[6] R. Otin, International Journal of Solids and Structures 50: 1605-1612 (2013)

[7] E. Paese et al., Applied Mathematical Modeling 39: 309-321 (2014)

[8] E. Thibaudeau et al., Journal of Materials Pro. Technology 215: 251-263 (2015)

[9] L. Langstädtler et al., Proceeding Paper of the 4M/ICOMM 2015 Conference (2015)

[10] K. Tracht et al., Micro Metal Forming, Editor: F. Vollertsen, Springer (2013) 